\title{
Perception of three-dimensional shape specified by optic flow by 8-week-old infants
}

\author{
MARTHA E. ARTERBERRY \\ Gettysburg College, Gettysburg, Pennsylvania \\ and \\ ALBERT YONAS \\ University of Minnesota, Minneapolis, Minnesota
}

\begin{abstract}
Sensitivity of 8-week-old infants to optical flow specifying the shape of a three-dimensional object was assessed. Infants viewed kinetic random-dot displays that specified three-dimensional cubes. The cubes were identical except for the presence or absence of an interior corner. Half of the infants viewed the full display. The other half viewed the central region of the displays, where the flow specifying the presence or absence of the corner differed. Infants in the full-view condition looked significantly longer to a novel cube than to the familiar cube following habituation. In contrast, infants in the partial-view condition looked equally to the novel and familiar cubes, ruling out the possibility that infants who viewed the full displays merely discriminated differences in motion in the central region of the two displays. These findings suggest that infants as young as 8 weeks perceive three-dimensional object shape from optic flow.
\end{abstract}

Perception of the world based on motion has occupied perceptual psychologists for a number of decades (see Braunstein, 1962, for a review). Pioneering work by Helmholtz (1909/1924) suggested that the differential motion of objects located at different distances was an effective cue to depth, and informal experiments conducted by Mach (1886/1959) may have been the first in which perception of structure from motion was explored. Wallach and O'Connell (1953) performed the first formal experiments on the perception of three-dimensional shape from motion, which they called the kinetic depth effect. In their work, they found that a static shadow of an object projected onto a screen was perceived as a flat twodimensional design. However, when the object rotated, the deforming contours were perceived accurately as a three-dimensional object. Gibson $(1950,1966)$ placed perception based on motion-carried information at the center of his new theory and highlighted its importance for veridical perception. Motion-carried information is viewed widely as an effective source of information for spatial layout. Moreover, those studying the development of perceptual processes have found that early in life, humans are responsive to motion-carried information. In the present study, we investigated 8-week-old infants' ability to

This research was supported by a Research and Professional Development Grant awarded to M.E.A. by Gettysburg College. A.Y. was supported by a grant from the National Institute of Health (HD-07151). The authors thank Bill Wilson and several anonymous reviewers for helpful comments on previous drafts. A version of this paper was presented at the meeting of the Association for Research in Vision and Ophthalmology, May 1995, Fort Lauderdale, FL. Correspondence can be addressed to M. E. Arterberry, Department of Psychology, Box 407, Gettysburg College, Gettysburg, PA 17325 (e-mail: arterber@gettysburg.edu). discriminate between patterns of motion that carry information for the three-dimensional shape of objects.

Research addressing infants' perception of depth and object shape specified by motion-carried information suggests that infants are able to utilize this information for perceiving spatial layout and three-dimensional object characteristics. For example, Kellman and Spelke (1983) showed that 4-month-old infants perceived the coherence of a partly occluded object if the visible ends of the object shared a common motion. Infants habituated to a horizontally moving rod whose center was occluded looked significantly longer to a fully visible broken rod than to a fully visible complete rod, suggesting that they perceived the partly occluded rod as a unified object. When the visible ends were stationary or moved in different directions, such as with rotation, the infants provided no evidence of perceiving the partly occluded object as a unified object.

In addition to perceiving object unity from motioncarried information, 5-month-olds, and possibly 3.5 montholds, segregate figure from ground on the basis of the common motion of object texture and object boundaries. Craton and Yonas (1990) presented infants with kinetic random dot displays that depicted a two-dimensional figure moving in front of a textured background. The only information for the boundary between figure and ground was the common motion between the texture on the surface of the figure and the figure boundary edge. Infants' pattern of looking suggested that they perceived the figure as a bounded region and that they perceived the background as an unbounded region continuing behind the figure.

Young infants also perceive spatial structure specified by motion-carried information. In several studies in which 4-month-old infants viewed rotating solid objects, rotat- 
ing wire figures, or kinetic random dot displays that simulated an oscillating object, infants provided evidence of perceiving the three-dimensional shapes of the objects. Infants looked significantly longer to a novel object than to a familiar object following habituation (Arterberry, 1992; Arterberry \& Yonas, 1988; Kellman, 1984; Kellman $\&$ Short, 1987). Furthermore, young infants perceive the location of a moving surface in depth. When presented with displays in which optic flow specifies a moving surface, 12-week-old, 6-week-old, and 3-week-old infants blink significantly more to an expanding flow field that specifies an approaching surface than to a contracting flow field that specifies a receding surface (Nanez, 1988; Nanez \& Yonas, 1994; Yonas, Pettersen, \& Lockman, 1979).

These studies provide a few examples of early sensitivity to motion-carried information by human infants. In 1966, Gibson described the way in which perspective transformation of the optic array-optic flow - can specify slants with greater specificity than can static perspective. For adults, optic flow may be generated by selfmotion and/or object motion. For young infants who are unable to locomote, active head motion, object motion, and/or passive motion may generate optic flow. People and objects in the infants' environment are frequently in motion, and infants move their heads and are often moved passively by locomoting others.

Motion thresholds for infants under 12 weeks of age, when they can be found, are typically high in comparison with adult thresholds (1-2 min arc/sec; Kaufman, 1974). Some researchers have had difficulty in identifying motion thresholds in infants 8 weeks and younger. For example, Volkmann and Dobson (1976) found little preference for horizontally moving displays by 4-weekolds, and Dannemiller and Freedland (1989) found no motion preferences at 8 weeks of age. Aslin and Shea (1990) found a 9-deg/sec threshold at 6 weeks and a 4$\mathrm{deg} / \mathrm{sec}$ threshold at 12 weeks for vertical motion. Thresholds for rotary motion were lower, 1.4 and $0.93 \mathrm{deg} / \mathrm{sec}$ for 1- and 3-month-olds, respectively; but these thresholds were still elevated in comparison with adult levels (Kaufmann, Stucki, \& Kaufmann-Hayoz, 1985), as were thresholds for oscillating gratings (Hamer \& Norcia, 1994). Wattam-Bell (1991, 1992, 1996a, 1996b) has identified changes in motion sensitivity between 6,8 , and 15 weeks of age. His work suggests that directional mechanisms are present by 8 weeks but that the velocity range over which they operate is very restricted.

In order to perceive the shape of an object from flow information, infants do not necessarily need to discriminate very slow motion from no motion; instead they need to be able to pick up gradients of velocity. As a three-dimensional object rotates, surface texture elements will move in the same direction but at different velocities. This relative motion among texture elements provides information for the orientation and location of the surfaces relative to one another. Recent research suggests differences in the developmental time course for different types of motions (see Banton \& Bertenthal, 1997 , for a review). Infants appear to respond to uniform motion, as in the case of translation, before they respond to relative motion, as in shear. For example, Banton and Bertenthal (1996) found little developmental differences in detection of uniform motion between 6,12 , and 18 weeks of age. In contrast, Bertenthal and Bradbury (1992) found that detection of shear improved between 13 and 20 weeks of age. In addition, there may be developmental changes in infants' detection of relative motion; Dannemiller and Freedland (1991) found that for 20-weekolds, detection of relative motion of elements moving in the same direction was less robust than the detection of the relative motions of elements moving in opposite directions. Similarly, Wattam-Bell (1994) found poor performance among 11- to 15-week-olds in discriminating coherent from noncoherent motions in which the texture elements within the coherent displays created stripes moving in opposite directions.

This study provides a test of 8-week-old infants' ability to detect optic flow that specifies the shape of a threedimensional object. Infants were presented with kinetic random dot displays, which specified for adults either a cube with a convex corner facing the viewer or an incomplete cube with a concave corner (Figures 1A and 1B). When the displays were stationary (Figures $\mathrm{IC}$ and 1D), they appeared as a random array of dots that did not specify any shape. When the displays moved, the relative motions of the dots specified a three-dimensional object. That is, the motion of the dots created the experience of planar tangible surfaces and all the dots were perceived as texture on those surfaces. In addition, the surfaces were perceived as intersecting with each other, creating sharp corners.

Previously, Arterberry and Yonas (1988) found that 16-week-old infants perceived three-dimensional object shape specified by the optic flow contained within these displays. In a habituation paradigm, 16-week-olds looked significantly longer to the novel display than to the familiar display. Moreover, when 16-week-olds viewed only the central region of the displays (see dotted lines in Figures $1 \mathrm{~A}$ and $1 \mathrm{~B}$, indicating the visible region), where differences in texture motion were most disparate between the convex and concave cubes, 16-week-old infants did not look significantly longer to the novel display than to the familiar display (Arterberry, 1992). This finding suggested that infants who were provided with a full view of the displays did not discriminate between them on the basis of these local motion differences.

In the present study, 8-week-old infants were presented with either a full view of the displays or with the central region of the displays (see dotted lines in Figures $1 \mathrm{~A}$ and $\mathrm{BB}$ ). The infants were habituated to either the convex or the concave cube oscillating about two different axes. Following habituation, the infants viewed both the convex and the concave cubes oscillating about a third new axis. Presenting infants with displays of objects rotating or oscillating around different axes was a 


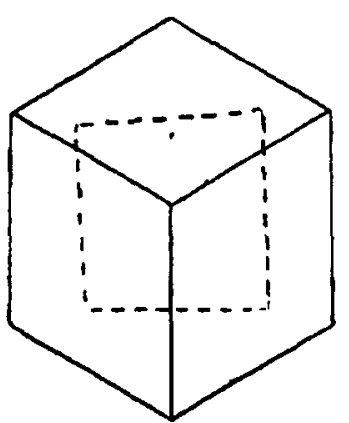

A

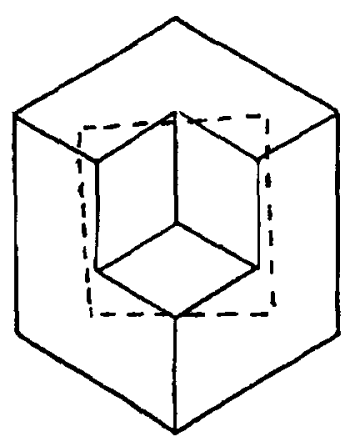

B

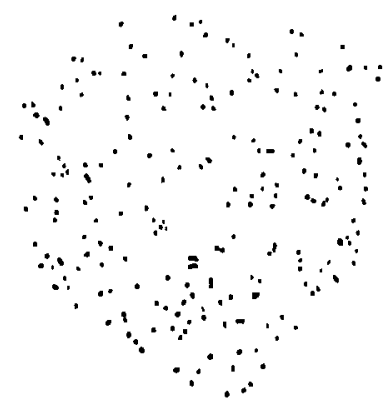

C

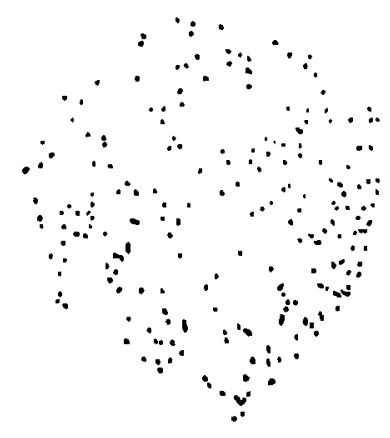

D

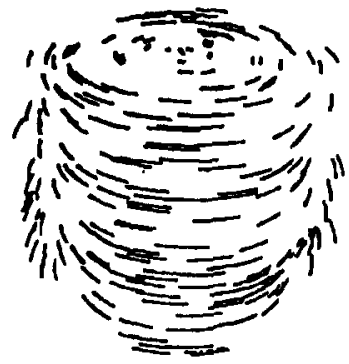

$\mathbf{E}$

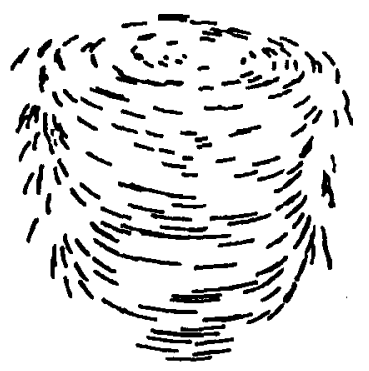

$\mathbf{F}$

Figure 1. Schematic drawings of the convex (A) and concave (B) cubes. These displays represent the middle frame of each oscillation. The dotted line in each drawing indicates the middle portion of the display that was visible through the aperture in the partial-view condition. (C) and (D) depict single frames of the convex and concave cubes. (E) and (F) were drawn from time-lapse photographs to show the pattern of texture flow in the convex and the concave cubes. In the actual displays, the contrast was reversed.

technique first used by Kellman (1984). In his study, infants viewed rotating solid three-dimensional forms. The reason for presenting the objects moving around a third new axis during the test phase stemmed from the concern that infants could respond to proximal information rather than distal shape. Significantly more looking to the novel object despite the fact that the familiar object was moving in a novel direction is a strong test of infants' three-dimensional shape perception; infants needed to recognize the familiar shape despite its new motion, and to attend longer to the new shape. The use of kinetic random dot displays makes it even less likely that infants could respond to proximal features of the displays rather than distal form, since figural information, such as surfaces and the intersections between them, is created by relative motion of the dots (e.g., Braunstein, 1962; Green, 1959). There is no information the in a single static view.

We predicted that if 8-week-old infants were sensitive to optir flow information specifying three-dimensional object shape, infants who were provided with the full displays would look significantly longer to the novel display than to the familiar display following habituation. In contrast, we predicted that infants who viewed only the central region of the displays would look equally to the novel and familiar test displays. This pattern of findings would suggest that infants viewing the full displays discriminated the novel and familiar cubes on the basis of differences in three-dimensional structure rather than differences in the velocity of the dots in the central regions of the displays.

\section{METHOD}

\section{Participants}

Twenty-four 8 -week-old infants (mean age $=59$ days, range $=$ 42-71 days) served as subjects. Half of the infants viewed the full display (full-view condition) and half viewed only the central region of each display (partial-view condition). An additional 22 infants began testing but did not complete the procedure because of fussiness (17) or because they fell asleep (5). Although this attrition rate may initially appear high, it is lower than the rate found by Arterberry and Yonas (1988), who used similar displays.

\section{Materials and Apparatus}

Six displays consisting of white dots on a black field were created using an Evans and Sutherland PS300 vector graphics terminal hosted by a VAX computer. The displays simulated an $11-\mathrm{cm}$ convex or concave cube undergoing a $30 \mathrm{deg}$ rotation around three axes: horizontal, vertical, and diagonal. The display perspective employed a viewing distance of $91.4 \mathrm{~cm}$. Schematic line drawings of the two display objects and the dot arrangements of a single frame of each display are shown in Figures 1A-1D. Figures $1 \mathrm{C}$ and ID represent the middle frame of the oscillation, the point at which the object had rotated $15 \mathrm{deg}$ of the $30 \mathrm{deg}$ oscillation. Note the dis- 
plays differ only in the presence of the convexity or concavity of the central trihedral vertex.

The displays were videotaped from the computer monitor and presented on a $50 \times 33 \mathrm{~cm}(31.0 \times 20.8 \mathrm{deg})$ video monitor. The ratio of the vertical and horizontal sizes of the computer monitor and the video monitor were the same. The dots filled a $25 \times 24 \mathrm{~cm}$ $(15.8 \times 15.2 \mathrm{deg})$ region in the center of the screen. Each display contained 240 dots, and the dot density was $1 / \mathrm{cm}^{2}$. Each dot was $.25 \mathrm{~cm}^{2}\left(.2 \mathrm{deg}^{2}\right)$. The background and dot luminances were approximately $.5 \mathrm{~cd} / \mathrm{m}^{2}$ and $68 \mathrm{~cd} / \mathrm{m}^{2}$, respectively. The dots forming the external edges of the cubes moved an average of $1.1 \mathrm{~cm} / \mathrm{sec}$ $(0.70 \mathrm{deg} / \mathrm{sec})$. The dots in the central region of the convex cube moved an average of $3.57 \mathrm{~cm} / \mathrm{sec}(2.27 \mathrm{deg} / \mathrm{sec})$. The dots in the central region of the concave cube moved an average of $0.83 \mathrm{~cm} / \mathrm{sec}$ $(0.53 \mathrm{deg} / \mathrm{sec})$.

Infants looked over a parent's shoulder or sat on a parent's lap $90 \mathrm{~cm}$ from the video monitor, which rested on a table. Side panels $(153 \times 74 \mathrm{~cm})$, resting on the floor and extending approximately $130 \mathrm{deg}$ from the table, restricted the infants' view of the rest of the room. Observers viewed the infants through $2-\mathrm{cm}$ holes in the side panels. Buttons connected to a laboratory microcomputer were pressed whenever the infant fixated the display. The computer recorded the infant's total fixation time on each trial. In addition, the computer signaled to the experimenters when each trial had ended and when the infant had reached the habituation criterion.

In the full-view condition, the infants viewed the monitor screen through a $37 \times 31 \mathrm{~cm}(23.2 \times 19.5 \mathrm{deg})$ aperture that allowed full view of the displays. In the partial-view condition, the infants viewed the monitor screen through a rectangular $13 \times 15 \mathrm{~cm}(8.3 \times$ $9.5 \mathrm{deg}$ ) aperture that allowed view of only the central region of the displays (see dotted regions in Figures $1 \mathrm{~A}$ and $\mathrm{lB}$ ). Both aperture panels (measuring $76 \times 51 \mathrm{~cm}, 45.8 \times 31.6 \mathrm{deg}$ ) were affixed to the monitor and were painted black. Between trials, a $76 \times 55$ $(45.8 \times 34.0 \mathrm{deg})$ black occluder was raised and lowered to hide or reveal the monitor screen.

\section{Procedure}

During the testing session, parents were instructed not to interact with their infants and were asked to wear opaque glasses so that they could not see the displays. Prior to the beginning of each trial, the occluder was lowered so that the monitor could not be seen. One experimenter turned on the videotape player to present a display. When the display was on the screen, the occluder was raised to initiate the trial. The habituation trials followed an infant-control procedure (Horowitz, Paden, Bhana, \& Self, 1972), continuing until the infant looked away from the display for $2 \mathrm{sec}$ or until $60 \mathrm{sec}$ had elapsed. Between trials, the screen was covered by the occluder and the tape was changed. After a 5-sec intertrial interval, another trial was initiated. This procedure was continued until the infant met the habituation criterion, which was defined as a $50 \%$ reduction in mean fixation time on 3 consecutive trials from the baseline (the mean of the first 3 trials). To be included in the baseline, the 1st trial had to be at least $10 \mathrm{sec}$ in duration. If infants did not reach the habituation criterion within 14 trials, the habituation phase was terminated ( 5 infants did not meet the criterion: 3 in the full-view condition, and 2 in the partial-view condition). When the habituation phase was completed, 4 test trials were presented, using the same infant-control procedure as in the habituation trials.

The infants were habituated, on alternate trials, to displays of the same object (e.g., the convex cube) oscillating about two axes (e.g., horizontal and vertical). Following habituation, the infants viewed on alternate trials displays of the convex and the concave cubes oscillating about a third new axis (e.g., diagonal). Habituation object (convex or concave cube) and order of test trials (novel first or familiar first) were counterbalanced across infants. Axes of oscillation presented in the habituation and test phases were randomly determined.
Two experimenters conducted the study. One experimenter recorded infant fixation times, and raised and lowered the occluder to initiate and terminate each trial. Because this experimenter did not know which display was being presented on a given trial or whether the infant was in the habituation or test phase of the experiment, her data were used in the analyses. The second experimenter controlled the video recorder and changed the tapes between trials. To obtain a measure of interjudge reliability, a third experimenter recorded the fixation times of 16 infants $(r=.83)$.

\section{RESULTS}

The infants' mean looking to the habituation and test displays is shown in Figures $2 \mathrm{~A}$ and $2 \mathrm{~B}$. Table 1 illustrates the mean looking times on the last six habituation trials as a function of condition and habituation object. Infants' looking on the last six habituation trials was analyzed in a $2 \times 2 \times 6$ analysis of variance (ANOVA) with condition (full view, partial view) and habituation object (convex cube, concave cube) as between-subjects factors and trial as a within-subjects factor. The analyses revealed a significant main effect of trial $[F(5,100)=6.25$, $p<.001]$, indicating that infants' looking times de-
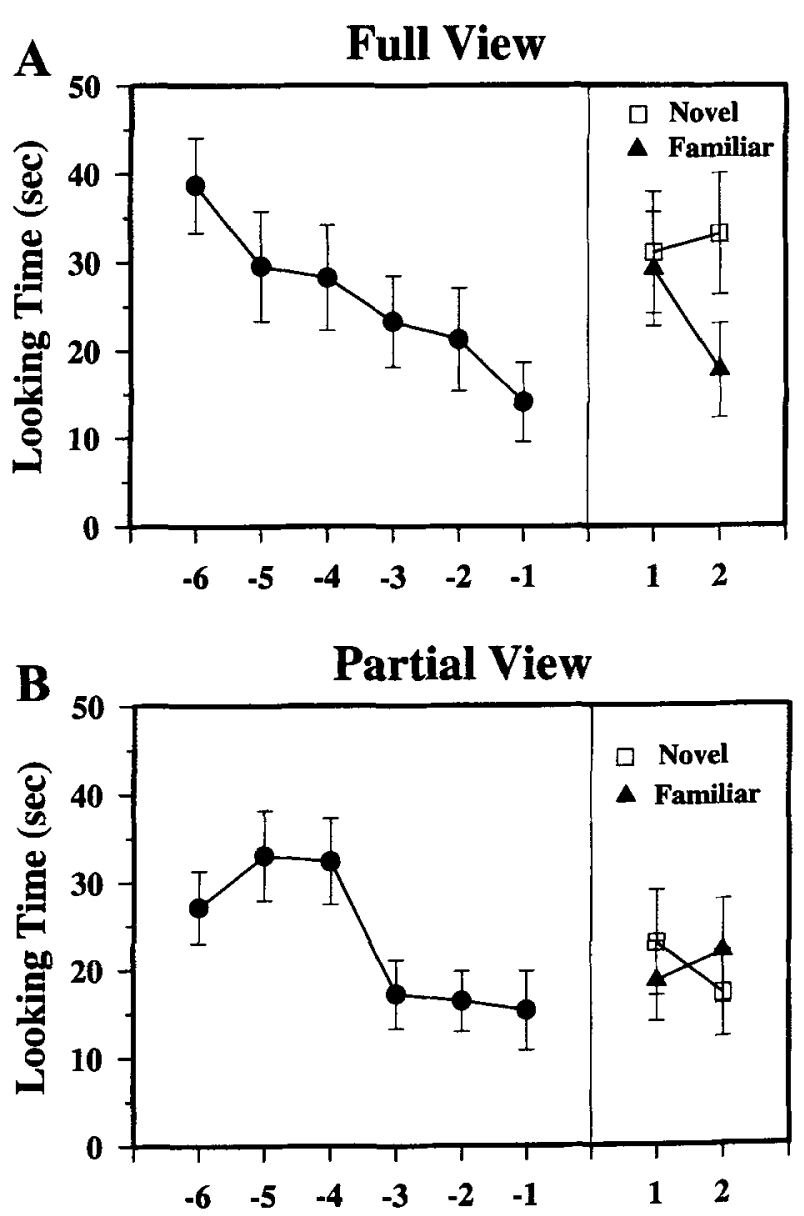

Figure 2. Mean fixation times during habituation and test trials for the full-view (A) and partial-view (B) conditions. 
Table 1

Infants' Looking to the Convex and the Concave Cubes and the Last Six Trials During the Habituation Phase in the Full-View and Partial-View Conditions

\begin{tabular}{llrrrrrr}
\hline & & \multicolumn{5}{c}{ Trials to Habituation } \\
\cline { 3 - 8 } Condition & & -6 & -5 & -4 & -3 & -2 & -1 \\
\hline Full View & & & & & & & \\
Convex cube & $M$ & 42.92 & 25.56 & 27.50 & 22.58 & 22.51 & 9.86 \\
& $S D$ & 16.39 & 26.66 & 19.53 & 20.01 & 22.03 & 8.69 \\
Concave cube & $M$ & 34.43 & 33.51 & 28.97 & 24.02 & 19.94 & 18.27 \\
& $S D$ & 21.11 & 16.24 & 23.47 & 17.47 & 20.39 & 19.94 \\
Partial View & & & & & & & \\
Convex cube & $M$ & 22.42 & 30.18 & 27.38 & 11.16 & 13.48 & 12.61 \\
& $S D$ & 12.43 & 16.52 & 18.29 & 8.38 & 8.69 & 12.61 \\
Concave cube & $M$ & 31.87 & 35.87 & 37.35 & 23.27 & 19.38 & 18.07 \\
& $S D$ & 15.89 & 19.73 & 15.34 & 15.65 & 14.74 & 20.22 \\
Total & $M$ & 32.91 & 31.28 & 30.30 & 20.26 & 18.83 & 14.70 \\
& $S D$ & 17.52 & 19.28 & 18.55 & 15.84 & 16.46 & 15.32 \\
\hline
\end{tabular}

creased across the last three trials (Tukey HSD $=12.81$, $p=.05$ ). No other significant main effects or interactions were found. A second 2 (condition) $\times 2$ (habituation object) ANOVA was conducted on the number of trials to reach the habituation criterion. These analyses revealed no significant main effects or interactions. The results of these analyses suggested that infants' looking time decreased across the habituation phase, as expected, and the amount of looking to the displays and the number of trials needed to reach the habituation criterion did not vary systematically between display presentations (full view or partial view) or between display types (convex or concave).

Preliminary analyses on the test trial data indicated no differences in amount of looking on the test trials by infants who did and did not reach the habituation criterion. Infants' looking times to the test displays were analyzed in a $2 \times 2 \times 2 \times 2 \times 2$ ANOVA with condition (full view, partial view), habituation object (convex cube, concave cube), and test order (novel first, familiar first) as betweensubjects factors and test display (novel, familiar) and trial as within-subjects factors. The analyses revealed a significant main effect of test display $[F(1,16)=5.18$, $p<.05]$, a condition $\times$ test display interaction $[F(1,16)=$ $5.66, p<.05]$, and a test order $\times$ condition $\times$ test display interaction $[F(1,16)=4.74, p<.05]$. No other significant main effects or interactions were found.

Tukey post hoc tests revealed that the condition $\times$ test display interaction was due to significantly more looking to the novel than to the familiar test display by infants in the full-view condition $[t(11)=2.94, p<.05]$. Infants in the partial-view condition looked equally to the novel and familiar test displays $[t(11)=0.44, p>.05]$. The three-way interaction with test display, condition, and test order was due to increased looking to the novel and familiar test displays as a function of testing order by infants in the partial-view group. Infants who viewed the novel display on the first test trial looked significantly longer to the novel than to the familiar test display, whereas infants who viewed the familiar display on the first test trial looked significantly longer to the familiar than to the novel test display $[t(11)=5.95, p<.01]$. The looking times of infants in the full-view condition did not vary as a function of test presentation order $[t(11)=$ $0.34, p>.05]$.

In summary, infants in the full-view condition looked significantly longer to the novel test display, which suggests that they perceived some aspect of three-dimensional structure in the kinetic random dot displays. Infants in the partial-view condition provided no evidence of perceiving three-dimensional shape. These infants looked significantly longer to the display presented first in the test phase, and it is likely that they were responding to differences in the direction of motion of the dots. Recall that the displays presented in the test phase oscillated around a third new axis, and as a result, the dots moved in a novel direction. Even though infants in the full-view condition also saw displays moving around a third new axis in the test phase, they were able to recognize the familiar object despite its new motion and attended longer to the novel object.

\section{DISCUSSION}

Eight-week-old infants provided evidence of perceiving the shape of three-dimensional objects specified by optic flow. Owing to the use of a partial-view condition, the possibility that young infants responded simply to velocity differences in the center of the displays can be ruled out. When presented with only the central region in the partial-view condition, infants did not look longer to the novel display than to the familiar display.

The finding of sensitivity to motion-carried information for three-dimensional object shape in infants younger than 4 months of age provides further support for the importance of this information in the perception of spatial layout. It appears that the very young infant who is not even able to crawl can make use of the motion of objects 
around him or her and the optical transformations generated by this motion. Within the first 2-3 months of life, infants perceive impending collision specified by optical expansion (Nanez, 1988; Nanez \& Yonas, 1994), and they discriminate between figure- and ground-specified boundary flow (Craton \& Yonas, 1990). The present study adds the possibility that infants perceive threedimensional shape specified by the relative motion of surface texture. The appearance of sensitivity to motioncarried information before the onset of stereopsis or sensitivity to pictorial depth cues further highlights the importance and usefulness of motion-carried information. It is possible that we enter the world ready to use this most reliable source of information (Kellman \& Arterberry, 1998).

Even though 8-week-olds appear to have limited motion sensitivity (e.g., Dannemiller \& Freedland, 1991; Wattam-Bell, 1992), they are able to use information provided by optic flow for perceiving the shapes of threedimensional objects. The results of this study suggest that very young infants' motion sensitivity may be better than has been previously thought. The motion contained in the displays used in this experiment ranged from 0.53 to $2.27 \mathrm{deg} / \mathrm{sec}$, which is much lower than the average estimates of motion thresholds for infants at this age. Von Hofsten, Kellman, and Putaansuu (1992) found similar improved performance in a study on observercontingent motion sensitivity. They found that 14-weekold infants showed sensitivity to a differential velocity of $.32 \mathrm{deg} / \mathrm{sec}$. It is possible that in the visual preference studies that have typically been used to assess infants' motion sensitivity, infant motion detection abilities may have been underestimated. When infants' sensitivity to motion-carried information is tested in the context of how it is normally used (i.e., in perception of the shape of objects), young infants appear to demonstrate greater competencies than when infants are asked to demonstrate a preference for slowly moving stimuli over static stimuli.

Some caution is needed in the interpretation of these results. It is exceedingly difficult to infer safely what 8week-old infants experience when they show discrimination of two displays. Whereas in this study we have ruled out the possibility that the infants discriminated between the displays solely on the basis of velocity differences, it is possible that they were able to detect the reversals in the sign of the gradient of velocity when these were available over the entire display. These reversals in the direction of the velocity gradient indicate to us that a form has a concave or convex corner facing the viewer. Yet it is possible that the infants perceived this higher order information without fully perceiving the shape that it specified. For example, the reversal in the sign of the velocity gradient that specifies a corner for adults may create for the infant some kind of subjective contour without fully generating a three-dimensional layout. If this were the case, the concave and convex displays would differ in the number of contours present. The convex display would generate three contours, whereas the concave display would generate six contours.

In the partial-view condition, the number of contours that were visible through the aperture were the same as in the full-view condition; the concave display generated six contours, and the convex display generated three contours. However, in the partial-view condition, the infants could not see the texture that extended beyond the internal contours nor the contours that were generated at the objects' external boundaries. When the aperture was present, adults reported that the velocity and direction of the dots were easily perceived; yet the display no longer supported the perception of surfaces and corners. When asked to judge the degree of similarity between two displays (two convex or two concave cubes) oscillating about two different axes, adults judged the two displays as less similar in the partial-view condition than in the full-view condition $[t(11)=2.45, p<.05]$. These results, in conjunction with the infants' performance in the partial-view condition, suggest that the motion of dots in the area surrounding the central region was necessary for the perception of corners to occur. It is likely that the deforming contours of the external boundaries of the full-view displays contributed to the perception of three-dimensional shape (Cortese \& Andersen, 1991; Norman \& Todd, 1994). Cortese and Andersen found that adults were able to determine the shape of ellipsoid silhouettes as they rotated. The only information for three-dimensional shape perception in their study was the deforming external contour. It seems likely that the reason the infants failed to discriminate between the displays in the partial-view condition was that in this condition information for the three-dimensional structure of the surfaces was reduced, as it is for adults.

A difference between the full-view and partial-view conditions was the size of the displays. The visible region was greatly reduced in the partial-view condition, and it might be possible that the infants failed to discriminate between the displays because they failed to attend to them, given their smaller size. Yet this possibility is unlikely, because the infants showed equally high levels of attention to the displays in both the full-view and the partial-view conditions during the habituation phase. Although the level of attention during the test phase was somewhat depressed in the partial-view condition as compared with the level of attention in the fullview condition, the distributions did overlap. In addition, infants in the partial-view condition looked to the test displays on average for 15 to $25 \mathrm{sec}$.

This study presents evidence of a remarkable ability in very young infants. At only 8 weeks of age, they show evidence of sensitivity to the higher order patterns of optic flow that specify the presence of surfaces, the orientation of those surfaces, and the locations of corners between those surfaces. Whereas 8 -week-old infants may not experience the layout of those surfaces in precisely the same way as adults would, infants are able to 
extract the information and use it to discriminate between displays.

\section{REFERENCES}

Arterberry, M. E. (1992). Infants' perception of three-dimensional shape specified by motion-carried information. Bulletin of the Psychonomic Society, 30, 337-339.

ARTERBERRY, M. E., \& YoNAS, A. (1988). Infants' sensitivity to kinetic information for three-dimensional object shape. Perception \& Psychophysics, 44, 1-6.

AsLin, R. N., \& SHEA, A. L. (1990). Velocity thresholds in human infants: Implications for the perception of motion. Developmental Psychology, 26, 589-598.

BANTON, T., \& Bertenthal, B. I. (1996). Infants' sensitivity to uniform motion. Vision Research, 36, 1633-1640.

Banton, T., \& Bertenthal, B. I. (1997). Multiple developmental pathways for motion processing. Optometry \& Vision Science, 74, 751-760.

Bertenthal, B. I., \& Bradbury, A. (1992). Infants' detection of shearing motion in random-dot displays. Developmental Psychology, 28, 1056-1066.

Braunstein, M. L. (1962). The perception of depth through motion. Psychological Bulletin, 59, 422-433.

Cortese, J. M., \& ANDERSEN, G. J. (1991). Recovery of 3-D shape from deforming contours. Perception \& Psychophysics, 49, 315-327.

Craton, L. G., \& Yonas, A. (1990). The role of motion in infants' perception of occlusion. In J. T. Enns (Ed.), The development of attention: Research and theory (pp. 21-46). Amsterdam: Elsevier, NorthHolland.

Dannemiller, J. L., \& Freedland, R. L. (1989). The detection of slow stimulus movement in 2- to 5-month-olds. Journal of Experimental Child Psychology, 47, 337-355.

Dannemiller, J. L., \& Freedland, R. L. (1991). Detection of relative motion by human infants. Developmental Psychology, 27, 67-78.

GIBSon, J. J. (1950). Perception of the visual world. Westport, CT: Greenwood Press.

GiBson, J. J. (1966). The senses considered as perceptual systems. Prospect Heights, IL: Waveland Press.

GreEN, B. F. (1959). Kinetic depth-effect (Psychology Group 58: Quarterly Progress Report). Cambridge, MA: Massachusetts Institute of Technology.

Hamer, R. D., \& Norcia, A. M. (1994). The development of motion sensitivity during the first year of life. Vision Research, 34, 2387 2402.

HelmHOLTZ, H. vON (1924). Helmholtz's physiological optics (3rd ed; J. P. C. Southall, Ed. \& Trans.). Rochester, NY: Optical Society of America. (Original work published 1909)

Horowitz, F. D., Paden, L. Bhana, K., \& Self, P. (1972). An infantcontrol procedure for studying infant fixations. Developmental Psychology, 7, 90.

KaufMAN, L. (1974). Sight and mind. New York: Oxford University Press.
Kaufmann, F., Stucki, M., \& Kaufmann-Hayoz, R. (1985). Development of infants' sensitivity for slow and rapid motions. Infant Behavior \& Development, 8, 89-98.

Kellman, P. J. (1984). Perception of three-dimensional form by human infants. Perception \& Psychophysics, 36, 353-358.

Kellman, P. J., \& ArTerberry, M. E. (1998). The cradle of knowledge. Development of perception in infancy. Boston: MIT Press.

Kellman, P. J., \& SHORT, K. (1987). Development of three-dimensional form perception. Journal of Experimental Psychology: Human Perception \& Performance, 13, 545-557.

Kellman, P. J., \& SPelKe, E. S. (1983). Perception of partly occluded objects in infancy. Cognitive Psychology, 15, 483-524.

$\mathrm{MACH}$, E. (1959). The analysis of sensations, and the relation of the physical to the psychical (C. S. Williams, Trans.; revised by S. Waterlow). New York: Dover. (Original work published 1886)

NANEZ, J. E. (1988). Perception of impending collision in 3- to 6week-old human infants. Infant Behavior \& Development, 11, 447463.

Nanez, J. E., \& Yonas, A. (1994). Effects of luminance and texture motion on infant defensive reactions to optical collision. Infant Behavior \& Development, 17, 165-174.

Norman, J. F., \& TODD, J. T. (1994). Perception of rigid motion in depth from the optical deformations of shadows and occlusion boundaries. Journal of Experimental Psychology: Human Perception \& Performance, 20, 343-356.

Volkmann, F. C., \& DoBson, M. V. (1976). Infant responses of ocular fixation to moving visual stimuli. Journal of Experimental Child Psychology, 22, 86-99.

von Hofsten, C., Kellman, P., \& Putaansuu, J. (1992). Young infants' sensitivity to motion parallax. Infant Behavior \& Development, 15, 245-264.

Wallach, H., \& O'ConNell, D. N. (1953). The kinetic depth effect. Journal of Experimental Psychology, 45, 205-217.

WATTAM-BELL, J. (1991). Development of motion specified cortical responses in infancy. Vision Research, 31, 287-297.

WATTAM-BELL, J. (1992). The development of maximum displacement limits for discrimination of motion direction. Vision Research, 32, $621-630$.

Wattam-Beli, J. (1994). Coherence thresholds for discrimination of motion direction in infants. Vision Research, 34, 877-883.

WATTAM-BELL, J. (1996a). Visual motion processing in one-monthold infants: Habituation experiments. Vision Research, 36, 1679 1685.

WatTAM-BeLL, J. (1996b). Visual motion processing in one-month-old infants: Preferential looking experiments. Vision Research, 36, 1671 1677.

Yonas, A., Pettersen, L., \& Lockman, J. (1979). Young infants' sensitivity to optical information for collision. Canadian Journal of Psychology, 53, 1285-1290.

(Manuscript received September 30, 1998; revision accepted for publication April 2, 1999.) 